\title{
ON DISCRETE BOREL SPACES AND PROJECTIVE SETS
}

\author{
BY B. V. RAO
}

Communicated by David Blackwell, December 3, 1968

Let $I$ denote the unit interval, $S=I \times I$ the unit square; $C_{I}$ and $C_{S}$ the class of all subsets of $I$ and $S$, respectively. By $C_{I} \times C_{I}$ is meant the $\sigma$-algebra on $S$ generated by rectangles with sides in $C_{I}$. The purpose of this note is to prove the following theorem (which settles a problem of S. M. Ulam) and observe some of its consequences. Withon:t explicit mention, the axiom of choice has been assumed throughout this paper. $\mathrm{CH}$ stands for the continuum hypothesis.

Theorem 1. If $C H$ is valid, then $C_{I} \times C_{I}=C_{S}$.

Proof. First, observe that if $f$ is any function defined on a subset of $I$ into $I$ then its graph

$$
G=\{(x, y): x \in \text { Domain of } f, f(x)=y\}
$$

is in $C_{I} \times C_{I}$. For this it suffices to verify that

$$
\begin{aligned}
G & =\bigcap_{n=1}^{\infty} S_{n} ; \text { where } S_{n}=\bigcup_{k=1}^{n}\left\{A_{n k} \times B_{n k}\right\}, \\
A_{n k} & =\{x \in \text { Domain } f:(k-1) / n \leqq f(x)<k / n\}, \\
B_{n k} & =\{y \in \text { Range } f:(k-1) / n \leqq y<k / n\} .
\end{aligned}
$$

(For $k=n$; include the right endpoint as well.)

Second, if $B C S$ be such that every vertical section is at most countable then $B \in C_{I} \times C_{I}$. This follows by realizing $B$ as countable union of graphs.

Third, if $B C S$ is such that every horizontal section is at most countable then $B \in C_{r} \times C_{r}$.

Fourth, $S=X \cup Y$ where every vertical section of $X$ is at most countable and every horizontal section of $Y$ is at most countable [4]. This can be done by realizing $I$ as the set of ordinals less than the first uncountable ordinal (by using $\mathrm{CH}$ ) and then taking the portions below and not below the diagonal.

Finally, if $B C S$ then by previous remarks $B \cap X, B \cap Y$ are in $C_{I} \times C_{I}$ to complete the proof.

Let $Z$ be a set of cardinality $N_{1}$, the first uncountable cardinal. An 
obvious modification of the above theorem gives us

THEOREM 2. The product of discrete $\sigma$-algebras on $Z$ is the discrete $\sigma$-algebra on $Z \times Z$. Consequently if $A \subset S$ be such that Card $(A) \leqq N_{1}$, then $A \in C_{I} \times C_{I}$.

Clearly, Theorem 1 is a consequence of the above theorem together with $\mathrm{CH}$.

ThEOREM 3. Let $\left\{Z_{\alpha}, \alpha \in T\right\}$ be any collection of subsets (possibly empty also) of $Z$ where Card $(T)=N_{1}$. Then there is a separable (countably generated and containing all singletons) $\sigma$-algebra on $Z$ containing the given collection.

Proof. There is no loss in taking $T=Z$, as we do. Put

$$
A=\bigcup_{\alpha \in Z}\left\{\{\alpha\} \times Z_{\alpha}\right\} .
$$

By Theorem 2, $A$ is in the product of discrete $\sigma$-algebras on $Z$ and consequently it is in the $\sigma$-algebra generated by a countable number of rectangles, say, $\left\{A_{i} \times B_{i}, i \geqq 1\right\}$. Any separable $\sigma$-algebra on $Z$ (clearly there are such) containing $\left\{A_{i}, B_{i}, i \geqq 1\right\}$ will suffice for our purpose.

As an immediate consequence of the above theorem we have the following which gives an affirmative answer to a question of S. M. Ulam [6], and disproves a conjecture of the author [2].

THEOREM 4. Let $\mathrm{CH}$ be valid. Then there is a separable $\sigma$-algebra on $I$ containing all the analytic sets of $I$. In fact there is one such containing all projective [3] sets of $I$.

In the terminology of Szpilrajn-Marczewski [1] the above theorem can be restated as

TheOREM 5. Let $\mathrm{CH}$ be valid. Then there is a one to one transformation $\phi$ of $I$ into $I$, transforming each set projective in I into a set Borel in $\phi(I)$.

We now formulate a generalization of the notion of projective sets and solve a related problem of Ulam [7]. Let $C=\left\{A_{p} ; p \in T\right\}$ be a collection of subsets of $I$ where Card $(T)=c$. The projections on $I$ of sets of the $\sigma$-algebra on $S$ over the rectangles $A_{p} \times A_{q}$ with sides in $C$, constitute $P_{1}$, the first projective class. Having defined $P_{\alpha}$ for $\alpha<\gamma<\Omega$ we define $P_{\gamma}$ as the projections on $I$ of the sets of the $\sigma$ algebra on $S$, over the rectangles with sides in the previous projective classes. These are called generalized projective sets. Clearly one 
need not proceed after the first uncountable ordinal $\Omega$. Since each $\boldsymbol{P}_{\boldsymbol{\alpha}}$ has cardinality not greater than $c$, we have

THEOREM 6. Let $\mathrm{CH}$ be valid. Then there is a separable $\sigma$-algebra on $I$ containing all its generalized projective sets over any fixed class $C$, where of course Card $(C) \leqq c$.

The author [2] has proved the following theorem, elsewhere.

ThEOREM 7. Let $L$ be any class of subsets of $I$ which are measurable w.r.t. a fixed nonatomic probability m?asure on the Borel subsets of $I$. Then $L$ does not contain any separable $\sigma$-algebra including all analytic subsets of $I$.

In view of Theorems 7 and 4, one has

THEOREM 8. Let $C H$ be valid. Fix any separable $\sigma$-algebra on I, say $\boldsymbol{A}_{0}$, containing all the analytic subsets of $I$. For every nonatomic probability measure on the Borel field of $I$, there is at least one nonmeasurable set in $\boldsymbol{A}_{0}$.

THEOREM 9. There exists a separable $\sigma$-algebra on $Z$ which supports no continuous probability measure.

Proof. Observe, following Ulam [5], that with each finite ordinal $n$ and countable ordinal $\alpha$ we can associate a subset $K(n, \alpha)$ of $Z$ satisfying the following:

(i) for each fixed $\alpha, \bigcup_{n} K(n, \alpha)$ is a cocountable subset of $Z$, and

(ii) for each fixed $n,\{K(n, \alpha): \alpha$ countable ordinal $\}$ is a disjoint family.

Take any separable $\sigma$-algebra on $Z$ containing all these sets (assured by Theorem 3). The argument of Ulam [5] now completes the proof.

If $\mathrm{CH}$ is assumed, the above theorem says that on $I$ there is a separable $\sigma$-algebra which does not support a continuous probability measure. If one wishes, this $\sigma$-algebra can be taken to contain all Borel sets or all analytic subsets of $I$.

The author expresses his appreciation and thanks to Dr. Ashok Maitra for the inspiring discussions and useful comments. Thanks are also due to him for many suggestions on an earlier version of this paper.

\section{REFERENCES}

1. E. Szpilrajn, The characteristic function of a sequence of sets and some of its applications, Fund. Math. 31 (1938), 222.

2. B. V. Rao, Remarks on analytic sets, Fund. Math. (to appear). 
3. W. Sierpinski, General topology, Univ. of Toronto Press, Ontario, 1952, p. 250.

4. - Cardinal and ordinal numbers, PWN, Warsaw, 1958, p. 376.

5. S. M. Ulam, Zur Masstheorie in der allgemeinen Mengenlehre, Fund. Math. 16 (1930), 140.

6. - Problèmes No. 74, Fund. Math. 30 (1938), 365.

7. - A collection of mathematical problems, Interscience, New York, 1960, p. 9.

Indian Statistical Institute, Calcutta, India

\section{ON SPHERE-BUNDLES. I}

BY I. M. JAMES ${ }^{1}$

Communicated by P. E. Thomas, November 19, 1968

Let $E$ be an $(n-1)$-sphere bundle over a base space $B$, with the orthogonal group as structural group. By an almost-complex structure on $E$ we mean a reduction of the structural group to the unitary group. By an $A$-structure on $E$ I mean a fibre-preserving map $f: E \rightarrow E$ such that $f x$ is orthogonal to $x$ for all $x \in E$. For example, an almostcomplex structure determines such a map through the action ${ }^{2}$ of the scalar $J$ such that $J^{2}=-1$. Note that $n$ must be even if an $A$-structure exists. When $E$ is trivial this necessary condition is also sufficient.

I describe $E$ as homotopy-symmetric if $1 \cong u: E \rightarrow E$, by a fibrepreserving homotopy, where $u$ denotes the antipodal map given by $u x=-x$. This condition also implies that $n$ is even. An $A$-structure $f$ on $E$ determines a fibre-preserving homotopy $f_{t}(t \in I=[0,1])$, where $f_{t} x=x \cos \pi t+f(x) \sin \pi t$, and so $E$ is homotopy-symmetric. I assert that the converse holds in the stable range ${ }^{3}$ so that we have

TheOREM 1. Let $B$ be a finite complex such that $\operatorname{dim} B \leqq n-4$. Then $E$ admits an $A$-structure if and only if $E$ is homotopy-symmetric.

A proof can be given as follows. Let $p: E \rightarrow B$ denote the fibration. Let $E^{\prime}$ denote the space of pairs $(x, y)$, where $x, y \in E$, such that $p x=p y$ and such that $x$ is orthogonal to $y$. We fibre $E^{\prime}$ over $E$ with projection $p^{\prime}$ given by $p^{\prime}(x, y)=x$. An $A$-structure $f$ on $E$ determines a cross-section $f^{\prime}: E \rightarrow E^{\prime}$, where $f^{\prime} x=(x, f x)$, and conversely a crosssection determines an $A$-structure. Let $E^{\prime \prime}$ denote the space of paths $\lambda$ in $E$ such that $p \lambda$ is stationary in $B$ and such that $\lambda(0)=\lambda(1)$. We

1 Research partly supported by the National Science Foundation.

2 We recall that the centre of the structural group acts on the bundle.

${ }^{3}$ The stable range, in relation to this problem, is not quite as extensive as the stable range of ordinary theory. 